\title{
Dermal fibroblasts, an old population in new environments
}

\author{
Wen Jie Zhang ${ }^{1}$, Wei Liu ${ }^{1}$, Lei Cui ${ }^{1}$, Guang Dong Zhou ${ }^{1}$, Yilin Cao ${ }^{1}$ \\ ${ }^{I}$ Department of Plastic and Reconstructive Surgery, Shanghai 9th People's Hospital, Shanghai Jiao Tong University School of \\ Medicine, National Tissue Engineering Center of China, Shanghai, China
}

Tissue engineering has become a new promising approach for repairing tissue defects and restoring tissue function in recent years. Based on the principle of tissue engineering, seeding cells play a crucial and indispensable role during the tissue regeneration process. Dermal fibroblasts are the major cell type in dermis and are commonly accepted as terminally differentiated cells. However, several reports demonstrated that dermal fibroblasts also present adipogenic, osteogenic, or chondrogenic potential. Unfortunately, there is yet no evidence that a single dermal fibroblast can differentiate into all these cell types. In the first study, we isolated dermal fibroblasts from human foreskin and clonal analyzed their adipogenic, osteogenic, and chondrogenic differentiation potential. Results from 47 clones showed that $6.4 \%$ of the clones were tri-potent, $19.1 \%$ of the clones were bi-potent, and $10.6 \%$ of the clones were single-potent, leaving $63.9 \%$ of the clones without any differentiation potential that have been tested, indicating that dermal fibroblasts are a heterogeneous population containing progenitors with different levels of differentiation potential. Furthermore, one of the three tri-potent clones exhibited neurogenic and hepatogenic differentiation potential. In the following study, we investigated whether dermal fibroblast could be utilized for tissue regeneration. Based on the idea that tissue specific environment plays an important role in cell fate determination, we utilized a corneal stroma-engineering model and demonstrated that dermal fibroblasts could switch their phenotype to corneal stroma cells in corneal environment. In addition, the implanted cells could functionally restore corneal transparency. Other examples have also been tested in tendon and cartilage regeneration. Dermal fibroblasts could successfully repair damaged tendon, but partially contribute to cartilage regeneration. Our work confirms the idea that in vivo niche is very important for cell fate determination and brings new insights into an old population. Further enrichment of multipotent cells from pooled population of dermal fibroblasts is under investigation.

Keywords: tissue engineering, dermal fibroblast, multipotency, cornea, tendon, cartilage

Cell Research (2008) 18:s114. doi: 10.1038/cr.2008.204; published online 4 August 2008

Correspondence: Wen Jie Zhang

E-mail: wenjieboshi@yahoo.com.cn

MD, PhD, Associate Professor.

Zhang graduated from Shanghai Jiao Tong University School of Medicine and got his MD degree in 1992. He did his PhD in Japan and took four years of post-doc training at Washington University School of
Medicine in St Louis. He worked with Dr Kyunghee CHOI on the early development o hematopoietic and endothelial cells utilizing an in vitro differentiation model of embryonic stem (ES) cells. He returned to China in 2004, and is now working on the differentiation of human ES cells. The goal of current work is to provide seed cells (such as endothelial cells, chondrocytes) from differentiated ES cells for tissue engineering. 